MZ-TH/99-07

CLNS/99-1606

hep-ph/9903412

March 1999

\title{
Configuration Space Based Recurrence Relations for Sunset-Type Diagrams
}

\author{
S. Groote ${ }^{1,2}$, J.G. Körner ${ }^{1}$ and A.A. Pivovarov ${ }^{1,3}$ \\ ${ }^{1}$ Institut für Physik, Johannes-Gutenberg-Universität, \\ Staudinger Weg 7, D-55099 Mainz, Germany \\ 2 Floyd R. Newman Laboratory of Nuclear Studies, \\ Cornell University, Ithaca, NY 14853, USA \\ ${ }^{3}$ Institute for Nuclear Research of the \\ Russian Academy of Sciences, Moscow 117312
}

\begin{abstract}
We derive recurrence relations for the calculation of multiloop sunset-type diagrams with large powers of massive propagators. The technique is formulated in configuration space and exploits the explicit form of the massive propagator raised to a given power. We write down and evaluate a convenient set of basis integrals. The method is well suited for a numerical evaluation of this class of diagrams. We give explicit analytical formulae for the basis integrals in the asymptotic regime.
\end{abstract}




\section{Introduction}

With the present accuracy of phenomenological applications, high precision tests of the Standard Model and search for new physics invariably require accounting for higher order contributions in coupling constants within perturbation theory. This necessitates the computation of multiloop diagrams [1]. Numerous high precision tests of the Standard Model require the evaluation of diagrams in the approximation when external momenta are small in comparison with the masses of particles corresponding to internal lines of the diagrams (see e.g. [2, 3, (4)). In this way massive diagrams without external momenta - vacuum bubbles - appear.

The dominant technique for high-order perturbation theory calculations of multiloop diagrams nowadays consists in using recurrence relations obtained from the method of integration by part within dimensional regularization [5]. The final answer of a calculation is expressed in terms of a few master integrals that serve as initial values for the recurrence equations. These master integrals are explicitly evaluated (analytically or numerically) while the reduction of a given diagram to the master integrals involves only algebraic manipulations (as an example see ref. [6] which presents the idea in its full bright glory).

Presently the evaluation of massive vacuum bubbles diagrams at the three-loop level is basically completed. The general strategy of reducing all massive three-loop bubbles to a basic set of master integrals through recurrence relations was described in ref. [7]. Recently the remaining unknown master integrals have been analytically identified by using high precision numerical computation assuming a given basis of transcendental numbers for master diagrams [8]. The existing techniques for the three-loop evaluation of massive vacuum bubbles make use of the reduction of six-dimensional objects (in the parameter space of powers of the propagators) through recurrence relations obtained within the integration by part technique. In direct applications of these techniques some topology classes of diagrams (sunsettype topology with large powers of propagators) appear to be one of the main sources of heavy computer time consumption [9]. Nevertheless, in principle, the problem of 
analytical calculation of three-loop bubbles is solved.

However, in practice the solution to the recurrence relations is not a routine procedure and requires the manipulation of a huge number of terms. Even using symbolic manipulation programs this task is in some cases beyond present computer capabilities. For instance, the computation of higher moments of the $b$-quark spectral function [10, 11], which is important for a precision determination of the $b$-quark mass [12], is limited by these obstacles and only moments up to $n=8$ are presently available within perturbation theory [13]. In view of the importance of this problem new ideas and techniques to improve on present results are called for.

Some attention has recently been drawn to the problem of optimizing the recurrence procedure for three-loop bubbles to find shorter routes to the final solution (see e.g. [14 and refs. therein). It has been proven that the final result for threeloop bubbles contains only several transcendental numbers (which are known) with rational coefficients for arbitrary mass configuration [8]. This structure of the final answer prompts one to search for more direct ways of obtaining the physical results. Indeed, for phenomenological applications one only needs numerical values of the coefficients of perturbation theory and their analytical expressions are not really important [2, 3]. The advantage of analytical computation is, of course, the full control over precision. If numerical methods are used, one has to take extra care about possible error accumulation. This is not always straightforward in this type of calculation because huge numerical cancellations may occur among the large number of contributing terms.

We discuss a technique that allows one to reduce these diagrams to a oneparameter set of basis integrals of a rather simple structure. The method is formulated in configuration space and exploits the explicit form of massive propagators with large powers of denominators. A convenient set of basis integrals is written down. The generalization to any number of loops is straightforward which is a part of the motivation for the present investigation. The method is well suited for a numerical evaluation of this class of diagrams.

In the course of comparing our analytical results with those given in ref. [6] 
we have derived a number of interesting new analytical results on definite integrals involving products of McDonald functions with powers and logarithms.

The paper is organized as follows. To begin with, in Sec. 2 we remind the reader of some features of the configuration space approach and introduce our notation which closely follows the notation in ref. [15]. In Sec. 3 we present several explicit examples taken from the subclass of sunset-type diagrams, the water melon diagrams, in order to compare the results obtained within the configuration space approach with results given in the literature [6]. In Sec. 4 we explain our general recursion concept and present a convenient set of basis integrals. In Sec. 5 we present different methods to calculate these basis integrals. Our conclusions are given in Sec. 6 .

\section{Basic relations}

We consider three-loop vacuum bubbles with only one mass $m$ which serves as a dimensional parameter. The classification of the topology prototypes for three-loop vacuum bubbles was presented in ref. [7]. The analytical computation of some missing master integrals has recently been completed [8]. However, the solution of the recurrence relations leading to the master integrals is complicated and time consuming, especially for large powers of propagators. We suggest new recurrence relations for a particular topology of vacuum bubbles which allows for an explicit solution. The simplicity of our technique is manifest in the configuration space representation for Feynman diagrams. First we remind the reader of some features of the configuration space approach and introduce our notation which closely follows the notation in ref. [15].

A general three-loop vacuum diagram has the topology of a tetrahedron. It also can be thought of as "fish+propagator" topology (see Fig. 1) where the "fish" part is a master two-loop diagram. This topology suggests the use of the following representation in configuration space (with an obvious choice of space-time points)

$$
\Pi(x)=\operatorname{fish}(x) D(x, m)
$$




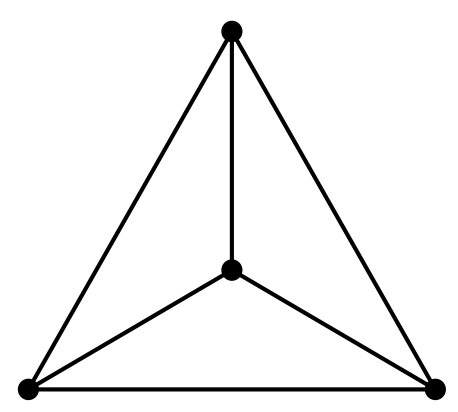

(a)

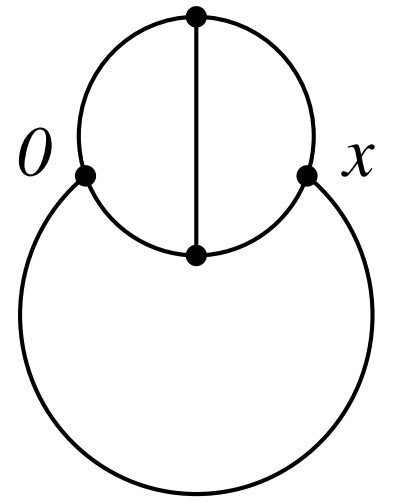

(b)

Figure 1: Three-loop vacuum bubble diagram in two different representations: (a) the tetrahedron representation, (b) the "fish+propagator" representation where the configuration space points 0 and $x$ are indicated.

where $D(x, m)$ is a propagator of a massive particle with mass $m$ in $D$-dimensional (Euclidean) space-time,

$$
D(x, m)=\frac{1}{(2 \pi)^{D}} \int \frac{e^{-i p_{\mu} x^{\mu}} d^{D} p}{p^{2}+m^{2}}=\frac{(m x)^{\lambda} K_{\lambda}(m x)}{(2 \pi)^{\lambda+1} x^{2 \lambda}}, \quad D(x, 0)=\frac{\Gamma(\lambda)}{4 \pi^{\lambda+1} x^{2 \lambda}} .
$$

We write $D=2 \lambda+2, \lambda=1-\varepsilon ; K_{\lambda}(z)$ is the McDonald function (a modified Bessel function of the third kind, see e.g. [16, 17]). The propagator $D(x, m)$ depends only on the length of the space-time vector $|x|=\sqrt{x_{\mu} x^{\mu}}$ for which we simply write $x$. The explicit representation for the modified internal line with mass $m$ (power of the massive propagator) is given by

$$
D^{(\mu)}(x, m)=\frac{1}{(2 \pi)^{D}} \int \frac{e^{-i p_{\nu} x^{\nu}} d^{D} p}{\left(p^{2}+m^{2}\right)^{\mu+1}}=\frac{1}{(2 \pi)^{\lambda+1} 2^{\mu} \Gamma(\mu+1)}\left(\frac{m}{x}\right)^{\lambda-\mu} K_{\lambda-\mu}(m x) .
$$

It contains the same functions (up to the difference in indices) as Eq. (2) and thus does not change the general functional structure of the representation constructed below. This is the reason why our method is well suited for dealing with large powers of propagators of massive particles.

The quantity of interest - a vacuum bubble - is represented by the integral

$$
\tilde{\Pi}(0)=\int \Pi(x) d^{D} x
$$


which is nothing but the Fourier transform of $\Pi(x)$ at zero momentum. It depends on a single dimensional parameter $m$, the mass of the particles in the massive lines.

The "fish" part of the diagram is written in terms of a dispersion relation in configuration space,

$$
\operatorname{fish}(x)=\int \rho_{f}(s) D(x, \sqrt{s}) d s
$$

which leads to a representation of the form

$$
\tilde{\Pi}(0)=\int \Pi(x) d^{D} x=\int d s \rho_{f}(s) \int D(x, \sqrt{s}) D(x, m) d^{D} x
$$

This form can be further simplified by performing the integration in $x$ explicitly. The result reads

$$
\begin{gathered}
\int D(x, \sqrt{s}) D(x, m) d^{D} x=\left(\frac{m \sqrt{s}}{2}\right)^{\lambda} \frac{1}{(2 \pi)^{\lambda+1} \Gamma(\lambda+1)} \int_{0}^{\infty} x K_{\lambda}(x \sqrt{s}) K_{\lambda}(m x) d x \\
=\left(\frac{m \sqrt{s}}{2}\right)^{\lambda} \frac{\pi}{(2 \pi)^{\lambda+1} \Gamma(\lambda+1)} \Gamma\left(\frac{1}{2}+\lambda\right) \Gamma\left(\frac{1}{2}-\lambda\right){ }_{2} F_{1}\left(\frac{1}{2}+\lambda, \frac{1}{2} ; 1 ; 1-\frac{m^{2}}{s}\right) .
\end{gathered}
$$

Here ${ }_{2} F_{1}(a, b ; c ; z)$ is a hypergeometric function. Therefore the problem of evaluating the diagram is reduced to a one-dimensional integral if the spectral density for the "fish" part $\rho_{f}(s)$ is known. The spectral density $\rho_{f}(s)$ was computed for the principal massive configurations in four-dimensional space-time [18]. If the propagator that multiplies the "fish" part of the diagram is massless, this formula can be further simplified and is given by

$$
\int D(x, \sqrt{s}) D(x, 0) d^{D} x=\frac{s^{\lambda / 2}}{2 \lambda(2 \pi)^{\lambda+1}} \int_{0}^{\infty} x^{1-\lambda} K_{\lambda}(x \sqrt{s}) d x=\left(\frac{s}{2}\right)^{\lambda / 2} \frac{\Gamma(1-\lambda)}{4 \lambda(2 \pi)^{\lambda+1}} .
$$

The final integration over $s$ in Eq. (6) now includes only some power of the energy square $s$ (instead of the hypergeometric function as in the massive case) and is rather straightforward. The equivalent set of formulae can also be obtained in the momentum space representation [8].

In some cases one propagator can be removed from a diagram using the recurrence relations for bubbles obtained within the integration by part technique [7]. Then the diagram becomes simpler. The initial "fish+propagator" topology converts to a "spectacles+propagator" topology. The spectral density for the spectacle 


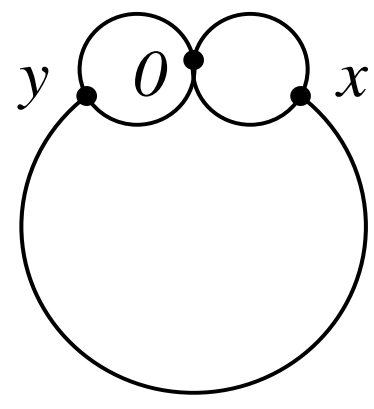

(a)

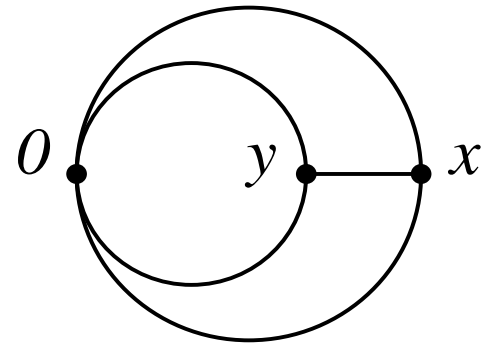

(b)

Figure 2: The "spectacle+propagator" representation, also called "spectacle" topology diagram in two different forms, namely (a) the form used in Eq. (69) and (b) the form used in Eq. (81).

part can be computed in a rather simple way. It is given by a product of two one-loop integrals in the momentum space representation (see Fig. 2). In the original classification of [7] these are class $E$ diagrams. We will henceforth adopt the classification of [7] to denote the different topology classes of diagrams.

After a deliberate use of the recurrence relations for bubbles, in some cases two propagators can be removed. A typical situation of such a kind was analyzed in ref. [6]. In such a case the diagrams become simple indeed. Even the most complicated ones belong to the subclass of water melon topologies which can be computed immediately (see Fig. 3). Their properties (with generalization to any number of loops) have been described in detail in ref. [15]. The most attractive feature of such a strategy is that for high derivatives of propagators (large powers of denominators) the corresponding recurrence relations for this particular topology can be solved very efficiently.

\section{Water melon topology: explicit examples}

While the water melon class of diagrams can appear as part of the remnants of the general recursive procedure, there are some cases when they are just the final aim 


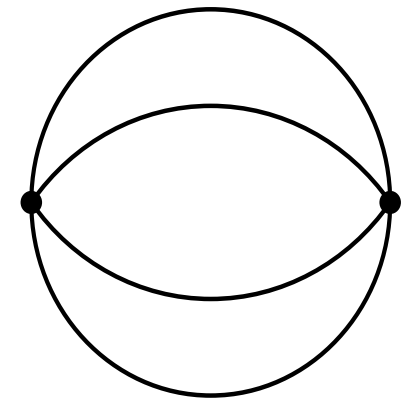

(a)

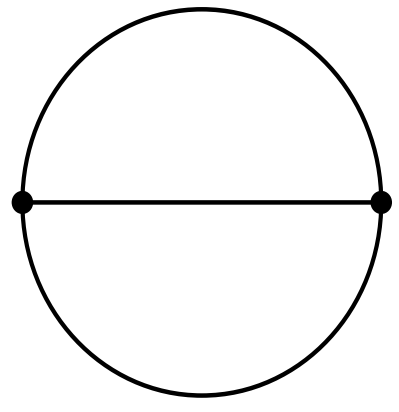

(b)

Figure 3: (a) Three-loop (i.e. four-line) water melon and (b) two-loop water melon (three-line water melon, being the ordinary sunset diagram).

of the recursion procedure. This is the case for the $B_{N}$ subclass of diagrams [6]. Some of the master integrals (for instance, $D_{3}(0,1,0,1,1,1)$ in ref. [7]) are exactly water melons. A further simplification of water melon diagrams can be achieved with the use of their particular properties. In the configuration space representation the water melon diagrams can be reduced to a specific basis set of simple integrals quite efficiently.

The configuration space technique for the water melon topology is particularly convenient for a numerical evaluation since it is simple and efficient. We have reproduced numerically some results of refs. [6, 8] with our technique.

In the particular case of the subclass $B_{N}$ of the bubble diagrams, the water melon topology diagrams emerge naturally and can be chosen as master configurations. The reduction of a general diagram of this subclass to the water melon topology is explicitly constructed in ref. [6]. Here we discuss a representation which can be obtained within the configuration space technique. The representation is simple and is given by a one-dimensional integral. The expansion in $\varepsilon$ within dimensional regularization is straightforward and is explicitly given for the evaluation of the numerical value of the integral $B_{4}$ [6] for which we give a new representation.

The starting point of our calculation is the definition of the $B_{N}$ class of dia- 
grams [6],

$$
\begin{aligned}
B_{N}\left(0,0, n_{3}, n_{4}, n_{5}, n_{6}\right) & =\int \frac{d^{D} k d^{D} l d^{D} p}{m^{3 D}\left(\pi^{D / 2} \Gamma(1+\varepsilon)\right)^{3}} \times \\
\frac{m^{2 n_{3}}}{\left((p+k)^{2}+m^{2}\right)^{n_{3}}} & \frac{m^{2 n_{4}}}{\left((p+l)^{2}+m^{2}\right)^{n_{4}}} \frac{m^{2 n_{5}}}{\left((p+k+l)^{2}+m^{2}\right)^{n_{5}}} \frac{m^{2 n_{6}}}{\left(p^{2}+m^{2}\right)^{n_{6}}}
\end{aligned}
$$

with two propagators absent $\left(n_{1}=n_{2}=0\right)$ to obtain a water melon topology for the three-loop case. In the following we will suppress these first two indices in the notation for the $B_{N}$ class diagrams. The configuration space expression for the generalized propagator (with crosses or having differentiated in its mass or momentum) is given by Eq. (3). This can be inserted into the above expression for $B_{N}$ and after rearrangement of integrations leads to

$$
\begin{aligned}
& B_{N}\left(n_{3}, n_{4}, n_{5}, n_{6}\right)=\frac{m^{2\left(n_{3}+n_{4}+n_{5}+n_{6}\right)-3 D}}{\left(\pi^{D / 2} \Gamma(1+\varepsilon)\right)^{3}} \times \\
& \quad(2 \pi)^{3 D} \int D^{\left(n_{3}-1\right)}(x, m) D^{\left(n_{4}-1\right)}(x, m) D^{\left(n_{5}-1\right)}(x, m) D^{\left(n_{6}-1\right)}(x, m) d^{D} x
\end{aligned}
$$

which can be reduced to a one-dimensional integral using the rotational invariance of the integration measure in Euclidean space-time,

$$
d^{D} x=\frac{2 \pi^{\lambda+1}}{\Gamma(\lambda+1)} x^{2 \lambda+1} d x .
$$

Note that various techniques of eliminating tensorial structures for vacuum diagrams were discussed in refs. 15, 19]. On the other hand we have [15]

$$
\begin{gathered}
\tilde{\Pi}(0)=\frac{2 \pi^{\lambda+1}}{\Gamma(\lambda+1)} \int_{0}^{\infty} D^{\left(n_{3}-1\right)}(x, m) D^{\left(n_{4}-1\right)}(x, m) \times \\
D^{\left(n_{5}-1\right)}(x, m) D^{\left(n_{6}-1\right)}(x, m) x^{2 \lambda+1} d x .
\end{gathered}
$$

The comparison of these two formulas results in

$$
B_{N}\left(n_{3}, n_{4}, n_{5}, n_{6}\right)=\frac{(2 \pi)^{3 D} m^{2\left(n_{3}+n_{4}+n_{5}+n_{6}\right)-3 D}}{\left(\pi^{D / 2} \Gamma(1+\varepsilon)\right)^{3}} \tilde{\Pi}(0)
$$

where the powers of the propagators in $\tilde{\Pi}(0)$ have been appropriately adjusted. In the following calculations we set $m=1$ for the mass (regardless of the units, of course). We will discuss some explicit applications of Eqs. (10) and (11) in the following to indicate their properties. 
As a first example we consider the integral $B_{N}(2,2,2,2)$ in the case $\lambda=1$ (fourdimensional space-time). Generally we have the explicit expressions

$$
D^{(1)}(x, 1)=\frac{x^{1-\lambda}}{(2 \pi)^{\lambda+1} 2^{1} \Gamma(2)} K_{\lambda-1}(x)=\frac{x^{\varepsilon}}{2(2 \pi)^{2-\varepsilon}} K_{-\varepsilon}(x)
$$

for the propagators which results in

$$
D^{(1)}(x, 1)=\frac{1}{2(2 \pi)^{2}} K_{0}(x)
$$

for $\varepsilon=0$. We obtain

$$
B_{N}(2,2,2,2)=\frac{(2 \pi)^{12}}{\pi^{6}} \frac{2 \pi^{2}}{16(2 \pi)^{8}} \int_{0}^{\infty} K_{0}^{4}(x) x^{3} d x=2 \int_{0}^{\infty} K_{0}^{4}(x) x^{3} d x
$$

which is the expression obtained earlier in 15. Note that the function $K_{0}(x)$ is a propagator of a massive particle in two-dimensional space-time. Therefore many results can be obtained by using two-dimensional field theory in Euclidean spacetime (see e.g. ref. 20]).

For the more general case of $D$-dimensional space-time we obtain

$$
\tilde{\Pi}(0)=\frac{2 \pi^{2-\varepsilon}}{16(2 \pi)^{8-4 \varepsilon} \Gamma(2-\varepsilon)} \int_{0}^{\infty} x^{4 \varepsilon} K_{-\varepsilon}^{4}(x) x^{3-2 \varepsilon} d x
$$

and

$$
B_{N}(2,2,2,2)=\frac{2^{1-2 \varepsilon}}{(1-\varepsilon) \Gamma(1+\varepsilon)^{3} \Gamma(1-\varepsilon)} \int_{0}^{\infty} K_{-\varepsilon}^{4}(x) x^{3+2 \varepsilon} d x .
$$

To find higher orders in the $\varepsilon$-expansion necessary for computations within dimensional regularization we use series expansions in $\varepsilon$ of all quantities entering Eq. (15). First we have the rather obvious results

$$
\begin{aligned}
\frac{2^{1-2 \varepsilon}}{(1-\varepsilon) \Gamma(1+\varepsilon)^{3} \Gamma(1-\varepsilon)} & =2\left(1+\varepsilon-2 \varepsilon \ln 2+2 \varepsilon \gamma_{E}\right)+O\left(\varepsilon^{2}\right), \\
x^{3+2 \varepsilon} & =x^{3}(1+2 \varepsilon \ln x)+O\left(\varepsilon^{2}\right),
\end{aligned}
$$

where $\gamma_{E}$ is Euler's constant. Within the dimensional regularization scheme the propagator in the configuration space contains the McDonald function with a noninteger index depending on the regularization parameter $\varepsilon$. To expand the McDonald function in the parameter $\varepsilon$ entering its index, we use the general formula 17

$$
\left[\frac{\partial K_{\nu}(z)}{\partial \nu}\right]_{\nu= \pm n}= \pm \frac{1}{2} n ! \sum_{k=0}^{n-1}\left(\frac{z}{2}\right)^{k-n} \frac{K_{k}(z)}{k !(n-k)}, \quad n \in\{0,1, \ldots\}
$$


for the derivative of the McDonald function with respect to its index near integer values of this index. In this case one obtains

$$
K_{-\varepsilon}(x)=K_{0}(x)+O\left(\varepsilon^{2}\right)
$$

We end up with

$$
\begin{aligned}
B_{N}(2,2,2,2)= & 2 \int_{0}^{\infty} K_{0}^{4}(x) x^{3} d x+2 \varepsilon\left(1+2 \ln 2+2 \gamma_{E}\right) \int_{0}^{\infty} K_{0}^{4}(x) x^{3} d x \\
& \quad+4 \varepsilon \int_{0}^{\infty} K_{0}^{4}(x) x^{3} \ln x d x+O\left(\varepsilon^{2}\right) \\
= & 2 I_{0}(3)+2 \varepsilon\left(1-2 \ln 2+2 \gamma_{E}\right) I_{0}(3)+4 \varepsilon I_{0}^{l}(3)+O\left(\varepsilon^{2}\right)
\end{aligned}
$$

where a general notation for the configuration space integrals

$$
\begin{aligned}
& I_{m}(q)=\int_{0}^{\infty} K_{0}^{4-m}(x) K_{1}^{m}(x) x^{q} d x \\
& I_{m}^{l}(q)=\int_{0}^{\infty} K_{0}^{4-m}(x) K_{1}^{m}(x) x^{q} \ln x d x
\end{aligned}
$$

is introduced. The only new contribution in the $\varepsilon$-expansion till the first order in Eq. (19) is connected with the logarithmic integral $I_{0}^{l}(3)\left(I_{m}^{l}(q)\right.$ from Eq. (20)). The part related to $I_{0}(3)$ in this order is a trivial kinematic contribution. The term $2\left(\ln 2-\gamma_{E}\right)$ in Eq. (19) can be easily removed by redefining the logarithmic integral using $\ln x \rightarrow \ln \left(x e^{\gamma_{E}} / 2\right)$.

Identifying the parameters $B_{3}$ and $B_{4}$ from [6] we find

$$
\begin{aligned}
B_{N}(2,2,2,2) & =-\frac{3}{8}+\frac{7}{16} B_{3}+\left(\frac{63}{32} B_{3}+\frac{3}{16} B_{4}\right) \varepsilon+O\left(\varepsilon^{2}\right) \\
& =-\frac{3}{8}+\frac{7}{16} \zeta(3)+\left(\frac{63}{32} \zeta(3)-\frac{63}{32} \zeta(4)+\frac{3}{16} B_{4}\right) \varepsilon+O\left(\varepsilon^{2}\right)
\end{aligned}
$$

where $B_{3}=\zeta(3)-\frac{9}{2} \varepsilon \zeta(4)+O\left(\varepsilon^{2}\right)$. The comparison of the zeroth order term of Eq. (21) with Eq. (19) results in the relation

$$
I_{0}(3)=-\frac{3}{16}+\frac{7}{32} \zeta(3)
$$

which assigns a value to one of the initial terms of the recurrence relations that will be presented later in Sec. 4. We checked this relation numerically. In the first order 
part of the $\varepsilon$-expansion we solve for $B_{4}$ obtaining the representation

$$
\begin{aligned}
B_{4} & =\frac{16}{3}\left(2\left(1-2 \ln 2+2 \gamma_{E}\right) I_{0}(3)+4 I_{0}^{l}(3)+\frac{63}{32}(\zeta(4)-\zeta(3))\right) \\
& =\frac{32}{3}\left(\left(1-2 \ln 2+2 \gamma_{E}\right) I_{0}(3)+2 I_{0}^{l}(3)\right)+\frac{21}{2}(\zeta(4)-\zeta(3))
\end{aligned}
$$

which after substituting for $I_{0}^{l}(3)$ from Eq. (20) gives numerically $B_{4}=-1.7628 \ldots$ This numerical value expressed in terms of configuration space integrals within our technique coincides with the result given in [6]. Taking the analytical expression for $B_{4}$ from [6]

$$
B_{4}=16 \operatorname{Li}_{4}\left(\frac{1}{2}\right)+\frac{2}{3} \ln ^{4}(2)-\frac{2}{3} \pi^{2} \ln ^{2}(2)-\frac{13}{180} \pi^{4}
$$

with $\mathrm{Li}_{4}(z)$ being a fourth order polylogarithm,

$$
\operatorname{Li}_{4}(z)=\sum_{k=1}^{\infty} \frac{z^{k}}{k^{4}}, \quad|z|<1
$$

we obtain the result for the logarithmic integral $\tilde{I}_{0}^{l}(3)$,

$$
\begin{aligned}
\tilde{I}_{0}^{l}(3) & =\int_{0}^{\infty} K_{0}^{4}(x) \ln \left(x e^{\gamma_{E}} / 2\right) x^{3} d x \\
& =\frac{3}{32}+\frac{3}{4} \operatorname{Li}_{4}\left(\frac{1}{2}\right)-\frac{17}{1920} \pi^{4}-\frac{1}{32} \pi^{2} \ln ^{2}(2)+\frac{1}{32} \ln ^{4}(2)+\frac{49}{128} \zeta(3)
\end{aligned}
$$

which serves as the initial value for the recurrence relations for the set of logarithmic integrals (Sec. 4). We have checked this value numerically. For a further demonstration of the efficiency of the configuration space technique for fixed powers of the propagators we calculate the two integrals $B_{N}(2,2,2,1)$ and $B_{N}(2,3,3,4)$ (the latter does not contain $B_{4}$ which is the reason for having selected this example).

For the integral $B_{N}(2,2,2,1)$ in four-dimensional space-time $(\lambda=1)$ we have to include the propagator

$$
D^{(0)}(x, 1)=\frac{x^{-\lambda}}{(2 \pi)^{\lambda+1} 2^{0} \Gamma(1)} K_{\lambda}(x)=\frac{x^{\varepsilon-1}}{(2 \pi)^{2-\varepsilon}} K_{1-\varepsilon}(x)
$$

equal to $K_{1}(x) / 4 \pi^{2} x$ for $\lambda=1$ which is a standard propagator of a massive particle for $D=4$. We obtain a representation of the form

$$
B_{N}(2,2,2,1)=4 \int K_{0}^{3}(x) K_{1}(x) x^{2} d x=4 I_{1}(2) .
$$


For the integral $B_{N}(2,2,2,1)$ in the case $\lambda=1-\varepsilon$ we obtain the generalization of Eq. (27) of the form

$$
B_{N}(2,2,2,1)=\frac{2^{2-2 \varepsilon}}{(1-\varepsilon) \Gamma(1+\varepsilon)^{3} \Gamma(1-\varepsilon)} \int_{0}^{\infty} K_{-\varepsilon}^{3}(x) K_{1-\varepsilon}(x) x^{2+2 \varepsilon} d x .
$$

The $\varepsilon$-expansion of the factor multiplying the integral is the same as in the former case except for an overall factor of 2 . A similar statement is valid for the expansion of the power of $x$. What remains to be done is to expand the McDonald functions in the vicinity of integer values of their indices. To obtain this expansion we use the relation

$$
\left.\frac{\partial K_{\nu}(x)}{\partial \nu}\right|_{\nu=1}=\frac{1}{2}\left(\frac{x}{2}\right)^{-1} K_{0}(x)=\frac{1}{x} K_{0}(x)
$$

which contributes to the power expansion as

$$
K_{1-\varepsilon}(x)=K_{1}(x)-\frac{\varepsilon}{x} K_{0}(x)+O\left(\varepsilon^{2}\right) .
$$

Using these expansions we obtain the representation in terms of our basic integrals

$$
\begin{aligned}
B_{N}(2,2,2,1)= & 4 \int_{0}^{\infty} x^{2} K_{0}^{3}(x) K_{1}(x) d x \\
& +4 \varepsilon\left(1-2 \ln 2+2 \gamma_{E}\right) \int_{0}^{\infty} x^{2} K_{0}^{3}(x) K_{1}(x) d x \\
& -4 \varepsilon \int_{0}^{\infty} x K_{0}^{4}(x) d x+8 \varepsilon \int_{0}^{\infty} x^{2} \ln x K_{0}^{3}(x) K_{1}(x) d x+O\left(\varepsilon^{2}\right) \\
= & 4 I_{1}(2)+4 \varepsilon\left(1-2 \ln 2+2 \gamma_{E}\right) I_{1}(2)-4 \varepsilon I_{0}(1)+8 \varepsilon I_{1}^{l}(2)+O\left(\varepsilon^{2}\right)
\end{aligned}
$$

that has to be compared with the output of the RECURSOR package [6] with the explicit master integrals $B_{3}, B_{4}$,

$$
\begin{aligned}
B_{N}(2,2,2,1) & =\frac{7}{4} B_{3}+\frac{3}{4} B_{4} \varepsilon+O\left(\varepsilon^{2}\right) \\
& =\frac{7}{4} \zeta(3)+\left(\frac{3}{4} B_{4}-\frac{63}{8} \zeta(4)\right) \varepsilon+O\left(\varepsilon^{2}\right)
\end{aligned}
$$

The zeroth order comparison gives the result $I_{1}(2)=\frac{7}{16} \zeta(3)$ which has been verified numerically. The first order comparison results in

$$
\begin{aligned}
B_{4} & =\frac{4}{3}\left(4\left(1-2 \ln 2+2 \gamma_{E}\right) I_{1}(2)-4 I_{0}(1)+8 I_{1}^{l}(2)+\frac{63}{8} \zeta(4)\right) \\
& =\frac{16}{3}\left(\left(1-2 \ln 2+2 \gamma_{E}\right) I_{1}(2)-I_{0}(1)+2 I_{1}^{l}(2)\right)+\frac{21}{2} \zeta(4)=-1.7628
\end{aligned}
$$


as before (see Eq. (23)). For the integral $B_{N}(2,3,3,4)$ in the case $\lambda=1-\varepsilon$ we need to include further propagators. They are

$$
\begin{aligned}
D^{(2)}(x, 1) & =\frac{x^{2-\lambda}}{(2 \pi)^{\lambda+1} 2^{2} \Gamma(3)} K_{\lambda-2}(x)=\frac{x^{1+\varepsilon}}{8(2 \pi)^{2-\varepsilon}} K_{-1-\varepsilon}(x), \\
D^{(3)}(x, 1) & =\frac{x^{3-\lambda}}{(2 \pi)^{\lambda+1} 2^{3} \Gamma(4)} K_{\lambda-3}(x)=\frac{x^{2+\varepsilon}}{48(2 \pi)^{2-\varepsilon}} K_{-2-\varepsilon}(x) .
\end{aligned}
$$

Both McDonald functions have to be expanded in their index. We have $K_{-1}(x)=$ $K_{1}(x)$ and

$$
\left.\frac{\partial K_{\nu}(x)}{\partial \nu}\right|_{\nu=-1}=-\frac{1}{2}\left(\frac{x}{2}\right)^{-1} K_{0}(x)=-\frac{1}{x} K_{0}(x)
$$

thus

$$
K_{-1-\varepsilon}(x)=K_{1}(x)+\frac{\varepsilon}{x} K_{0}(x)+O\left(\varepsilon^{2}\right),
$$

and

$$
\begin{aligned}
\left.\frac{\partial K_{\nu}(x)}{\partial \nu}\right|_{\nu=-2} & =-\frac{1}{2} 2 !\left(\frac{1}{2 !}\left(\frac{x}{2}\right)^{-2} K_{0}(x)+\frac{1}{1 ! 1 !}\left(\frac{x}{2}\right)^{-1} K_{1}(x)\right) \\
& =-\frac{2}{x^{2}} K_{0}(x)-\frac{2}{x} K_{1}(x),
\end{aligned}
$$

so that

$$
K_{-2-\varepsilon}=K_{2}(x)+\frac{2 \varepsilon}{x} K_{1}(x)+\frac{2 \varepsilon}{x^{2}} K_{0}(x)+O\left(\varepsilon^{2}\right) .
$$

With these relations we obtain the following $\varepsilon$-expansion for the integral in question

$$
\begin{aligned}
B_{N}(2,3,3,4)= & \frac{2^{-2 \varepsilon}}{192(1-\varepsilon) \Gamma(1+\varepsilon)^{3} \Gamma(1-\varepsilon)} \times \\
= & \frac{1}{192} I_{21}^{\infty} K_{-\varepsilon}(x)+\frac{\varepsilon}{192}\left(1-2 \ln 2+2 \gamma_{E}\right) I_{21}(7)+\frac{\varepsilon}{96} I_{11}(6) \\
& +\frac{\varepsilon}{96} I_{3}(6)+\frac{\varepsilon}{96} I_{2}(5)+\frac{\varepsilon}{96} I_{21}^{l}(7)+O\left(\varepsilon^{2}\right)
\end{aligned}
$$

where we have introduced the generalized integral

$$
I_{m n}(q)=\int_{0}^{\infty} K_{0}^{4-m-n}(x) K_{1}^{m}(x) K_{2}^{n}(x) x^{q} d x .
$$

This expansion has to be compared with the representation through master integrals found in momentum space,

$$
B_{N}(2,3,3,4)=\frac{1}{576}+\left(\frac{385}{65536} B_{3}-\frac{809}{884736}\right) \varepsilon+O\left(\varepsilon^{2}\right)
$$


resulting in the identification

$$
I_{21}(7)=\int_{0}^{\infty} K_{0}(x) K_{1}^{2}(x) K_{2}(x) x^{7} d x=\frac{1}{3}
$$

which is surprisingly simple and contains no transcendental numbers usually present in such integrals. It is rather curious that a similar identification allows one to express $\zeta(3)$ in terms of our basis integrals

$$
\begin{aligned}
\zeta(3)= & \frac{1024}{1155}\left(\left(1-2 \ln 2+2 \gamma_{E}\right) I_{21}(7)+2 I_{11}(6)\right. \\
& \left.+2 I_{3}(6)+2 I_{2}(5)+2 I_{21}^{l}(7)+\frac{809}{4608}\right) .
\end{aligned}
$$

We checked both equations numerically to make certain that they are valid. These results serve as a hint that the standard basis may not be the simplest and most relevant basis to be used in computations of massive three-loop diagrams.

We have considered some explicit expressions following from the configuration space representation of water melon diagrams. In all these examples the integrals are ultraviolet finite. We emphasize that ultraviolet divergences add nothing new to the analysis. For this particular topology the structure of the ultraviolet divergences is particularly simple: all divergences result from the region of integration around small $x$ and there are no overlapping divergences. Therefore the divergent parts of the diagrams can be obtained by expanding the integrand at small $x$ and subtracting the corresponding singularities which simplifies the integrands drastically (making them effectively massless) and the integration of the divergent parts can be done

analytically 15 within some (usually dimensional) regularization scheme. More complicated is to find the finite parts of the massive diagrams. Keeping this in mind we do not discuss the problem of ultraviolet divergences in the rest of the paper and concentrate on the finite parts.

\section{Reduction}

The preceding section has shown that three-loop water melon diagrams can be expressed as configuration space integrals of a product of at most four McDonald 
functions $K_{\nu}(x)$ where $\nu$ need not be an integer. In this section we want to exhibit the three steps of how to reduce the set of necessary integrals to a smaller set. First we can get rid of the non-integer dimensionality of the functions by using Eq. (17) to expand into powers of $\varepsilon$ resulting in integrals containing a product of four McDonald functions with or without a factor $\ln (x)$. As a second step we use the relation

$$
K_{n}(x)=2 \frac{n-1}{x} K_{n-1}(x)+K_{n-2}(x)
$$

for McDonald functions of different orders to further reduce the integrals to integrals only containing $K_{0}^{4}, K_{0}^{3} K_{1}, K_{0}^{2} K_{1}^{2}, K_{0} K_{1}^{3}$ and $K_{1}^{4}$ together with some positive powers of $x$ and again with or without a factor $\ln (x)$. The last step consists in using

$$
\frac{d}{d x} K_{\nu}(x)=-\frac{1}{2}\left(K_{\nu-1}(x)+K_{\nu+1}(x)\right)
$$

for integer $\nu$ (which is valid for any complex $\nu$ as well) and a partial integration in order to reduce the necessary integrals to integrals containing only the McDonald function $K_{0}(x)$. This reduction procedure will be considered for two different cases in the following two subsections.

\subsection{Reduction to integrals containing $K_{0}^{4}(x)$}

After using the recurrence relations for Bessel functions the general water melon diagram reduces to a linear combination of basic integrals given in Eq. (20). The subsequent reduction to even simpler integrals containing only $K_{0}^{4}(x)$ and powers of $x$ is done by partial integration. For this purpose we make use of the relations

$$
\begin{aligned}
\frac{d}{d x} K_{0}(x) & =-K_{1}(x) \quad \text { and } \\
\frac{d}{d x} K_{1}(x) & =-\frac{1}{2}\left(K_{0}(x)+K_{2}(x)\right)=-K_{0}(x)-\frac{1}{x} K_{1}(x) .
\end{aligned}
$$

After some simple algebra for the most tedious case of four functions $K_{1}(x)$ in the integrand we obtain the recursion relation

$$
\begin{aligned}
\int_{0}^{\infty} & K_{1}^{4}(x) x^{q} d x=-\int_{0}^{\infty} \frac{d}{d x}\left(K_{0}(x)\right) K_{1}(x)^{3} x^{q} d x \\
= & -\left[K_{0}(x) K_{1}^{3}(x) x^{q}\right]_{0}^{\infty} \\
& -3 \int_{0}^{\infty} K_{0}^{2}(x) K_{1}^{2}(x) x^{q} d x+(q-3) \int_{0}^{\infty} K_{0}(x) K_{1}^{3}(x) x^{q-1} d x .
\end{aligned}
$$


The other cases are simpler and will not be written down here. For $q>m$, the surface terms of the form $\left[K_{0}^{(4-m)}(x) K_{1}^{m}(x) x^{q}\right]_{0}^{\infty}$ vanish. Therefore the only elements of this recursion are the integrals $I_{m}(q)$, and the recursion is expressed as

$$
\begin{aligned}
I_{4}(q) & =(q-3) I_{3}(q-1)-3 I_{2}(q), \\
I_{3}(q) & =\frac{1}{2}\left((q-2) I_{2}(q-1)-2 I_{1}(q)\right), \\
I_{2}(q) & =\frac{1}{3}\left((q-1) I_{1}(q-1)-I_{0}(q)\right), \\
I_{1}(q) & =\frac{1}{4} q I_{0}(q-1)
\end{aligned}
$$

which reduces the starting integrals to our basis integrals

$$
I_{0}(q)=\int_{0}^{\infty} K_{0}^{4}(x) x^{q} d x
$$

\subsection{Reduction to integrals containing $K_{0}^{4}(x) \ln x$}

We again use the relations (41) to reduce the general water melon integrals to those containing only the McDonald function $K_{0}(x)$. This is done again by partial integration. As in the previous subsection we find

$$
\begin{aligned}
\int_{0}^{\infty} & K_{1}^{4}(x) x^{q} \ln x d x=-\int_{0}^{\infty} K_{1}^{3}(x) \frac{d K_{0}(x)}{d x} x^{q} \ln x d x \\
= & -\left[K_{0}(x) K_{1}^{3}(x) x^{q} \ln x\right]_{0}^{\infty}-3 \int_{0}^{\infty} K_{0}^{2}(x) K_{1}^{2}(x) x^{q} \ln x d x \\
& +(q-3) \int_{0}^{\infty} K_{0}(x) K_{1}^{3}(x) x^{q-1} \ln x d x+\int_{0}^{\infty} K_{0}(x) K_{1}^{3}(x) x^{q-1} d x
\end{aligned}
$$

for the case with four functions $K_{1}(x)$ in the integrand. For integer $q>m$, the surface terms $\left[K_{0}^{4-m}(x) K_{1}^{m}(x) x^{q} \ln x\right]_{0}^{\infty}$ again vanish. Therefore the recursion is expressed in terms of the integrals $I_{m}^{l}(q)$ and is given by

$$
\begin{aligned}
I_{4}^{l}(q) & =(q-3) I_{3}^{l}(q-1)+I_{3}(q-1)-3 I_{2}^{l}(q), \\
I_{3}^{l}(q) & =\frac{1}{2}\left((q-2) I_{2}^{l}(q-1)+I_{2}(q-1)-2 I_{1}^{l}(q)\right), \\
I_{2}^{l}(q) & =\frac{1}{3}\left((q-1) I_{1}^{l}(q-1)+I_{1}(q-1)-I_{0}^{l}(q)\right), \\
I_{1}^{l}(q) & =\frac{1}{4}\left(q I_{0}^{l}(q-1)+I_{0}(q-1)\right) .
\end{aligned}
$$


Together with Eqs. (42) these relations give the complete set of one-parameter recurrence equations for reducing a general water melon integral to a set of master integrals of the form $I_{0}(q)$ from Eq. (43) and

$$
I_{0}^{l}(q)=\int_{0}^{\infty} K_{0}^{4}(x) x^{q} \ln x d x
$$

Concluding these two subsections we affirm that Eqs. (43) and (45) constitute our basis for the evaluation of water melon diagrams with high powers of denominators.

\subsection{Efficiency of reduction}

Note that the direct reduction of a water melon diagram to the master integrals is rather slow within the straightforward application of recurrence relations based on momentum space representation. In practice the computation proceeds through the use of a table of integrals with given powers of the denominators. One would have a three-dimensional table for a given total power $N$ if no modifications of the basic technique as developed in ref. [6] have been introduced. The number of entries (even when accounting for the appropriate symmetries) then grows as fast as $N^{3}$ which is large for the large values of $N$ needed in some present applications. Within our methods one first re-expresses these integrals through a one-parameter set of integrals which are solved explicitly. For large $N$ the number of entries increases as

a first power of $N$ (the number of elements for the $I_{0}(q)$ basis is given by $2[N / 2]-5$ where $[z]$ is an integer part of $z$ ) which considerably reduces the time consumption in a computer evaluation. Note that in refs. [21, 22] different recursion techniques have been described which also avoid the use of the three-dimensional tables in reducing the water-melon diagram. For instance, in the package MATAD 21] the three-loop water melon diagrams are reduced to a one-dimensional table of integrals using a dedicated set of (momentum-based) recurrence relations. 


\section{Computation}

In this section we give some explicit formulae for computing water melon diagrams within the configuration space technique. The methods is best used for direct numerical computation or for constructing efficient approximation formulae for water melon diagrams. Therefore, as for exact analytical expressions, we mainly consider the $D=4$ case where the initial values of the recurrence procedure can be easily found and can be compared with numerical results. The construction of the $\varepsilon$-expansion is not drastically simplified as compared to the standard momentum space approach and we therefore do not dwell on it here.

\section{1 $N=4$ water melon}

We describe a simple way of computing an initial value of a general $D=4$ (or, more precisely, integer dimension) water melon diagram. The quantity we need has the form

$$
I(q)=\int_{0}^{\infty} K_{0}^{4}(x) x^{2 q+1} d x \equiv I_{0}(2 q+1)
$$

and represents a member of our basis.

We concentrate first on the case $q=0$. Note that Eq. (46) is simply the result for a water melon diagram with a massive propagator $K_{0}(m x)$ within a two-dimensional theory [20]. The corresponding two-line water melon (master one-loop diagram) in momentum space has the explicit form

$$
\tilde{\Pi}_{2}(p)=\frac{1}{2 \pi \sqrt{p^{2}} \sqrt{p^{2}+4 m^{2}}} \ln \left(\frac{\sqrt{p^{2}+4 m^{2}}+\sqrt{p^{2}}}{\sqrt{p^{2}+4 m^{2}}-\sqrt{p^{2}}}\right) .
$$

Eq. (46) now becomes

$$
I(0)=2 \pi m^{2} \int \tilde{\Pi}_{2}(p)^{2} d^{2} p=\frac{1}{4} \int_{0}^{\infty} \frac{\xi^{2} d \xi}{\sinh \xi}=\frac{7}{8} \zeta(3)
$$

where we have changed the integration variable $p$ to a new variable $\xi$ defined by the relation $p=2 m \sinh (\xi / 2)$ and used the standard integral 23

$$
\int_{0}^{\infty} \frac{\xi^{\alpha-1} d \xi}{\sinh \xi}=\frac{2^{\alpha}-1}{2^{\alpha-1}} \Gamma(\alpha) \zeta(\alpha)
$$


with $\Gamma(\alpha)$ being Euler's $\Gamma$-function and $\zeta(\alpha)$ being Riemann's $\zeta$-function. Note that the two-dimensional one-loop correlator in momentum space $\tilde{\Pi}_{2}$ shown in Eq. (47) has the simple form

$$
\tilde{\Pi}_{2}(2 m \sinh (\xi / 2))=\frac{1}{4 \pi m^{2}} \frac{\xi}{\sinh \xi}
$$

when expressed in terms of the new variable $\xi$. The integration measure becomes $d^{2} p=4 \pi m^{2} \sinh \xi d \xi$.

Results for other values of $q$ can be obtained by differentiating one of the two $\tilde{\Pi}_{2}$ in the integrand in Eq. (48),

$$
I(q)=2 \pi m^{2} \int \tilde{\Pi}_{2}(p)\left(-m^{2} \square_{p}\right)^{q} \tilde{\Pi}_{2}(p) d^{2} p
$$

where $\square_{p}$ is a two-dimensional d'Alembert operator in (Euclidean) momentum space, $\square_{p}=\partial^{2} / \partial p_{\mu} \partial p_{\mu}$. There is a possibility to differentiate a separate line of this three-loop water melon diagram that leads to different representations for higher moments but we find Eq. (50) to be the most convenient one. We do not have a general analytical solution to Eq. (50) for arbitrary large $q$ at the moment, although the solutions for some first values of $q$ are easily available.

Also we comment on the general structure of the basis given by $I(q)$ in Eq. (46). This basis has the form

$$
A_{q} \zeta(3)-B_{q}
$$

where the transcendental number $\zeta(3)$ is manifestly written down while $A_{q}, B_{q}$ are rational positive numbers for any $q$. Several pairs of coefficients $\left(A_{q}, B_{q}\right)$ for $q=1,2,3,4,5$ are

$$
\left(\frac{7}{32}, \frac{3}{16}\right), \quad\left(\frac{49}{128}, \frac{27}{64}\right), \quad\left(\frac{63}{32}, \frac{37}{16}\right), \quad\left(\frac{42777}{2048}, \frac{25555}{1024}\right), \quad\left(\frac{3101175}{8192}, \frac{9304913}{20480}\right) .
$$

The quantity $I(q)$ is positive for any $q$. Its numerical magnitude can be easily inferred from the asymptotic expansion of the integral given in Eq. (46) at large $q$,

$$
I(q)=\frac{\pi^{2} \Gamma(2 q)}{4^{2 q+1}}\left(1-\frac{1}{q-1 / 2}+O\left(1 / q^{2}\right)\right) .
$$

However, its analytical representation given in Eq. (51) reveals a rather awkward behaviour when analyzed numerically. Two terms with manifestly different transcendental structure taken as analytical expressions cancel each other to a large 
extent upon numerical evaluation. Indeed, using the numerical value of the transcendental number $\zeta(3)=1.20206 \ldots$, the numerical values of the pairs $\left(A_{q} \zeta(3), B_{q}\right)$ for $q=1,2,3,4,5$ are given by

$$
(0.26295,0.1875), \quad(0.460162,0.421875), \quad(2.36655,2.3125)
$$

For $q=5$ the numerical values of quantities of different transcendentality coincide with each other up to three significant figures resulting in a cancellation on subtraction and a large loss of precision. For larger $q$ the cancellation of the first significant figures of the two numbers is more dramatic resulting in even larger losses of numerical precision. It casts some doubt on whether the presentation of results in terms of their manifest transcendental structure is the most preferable one. On the other hand the asymptotic formula Eq. (52) is simple and quite precise. Namely, taking Eq. (52) and introducing a new parameter $\kappa$ which accounts for higher order terms in the asymptotic expansion, one has

$$
I(q)=\frac{\pi^{2} \Gamma(2 q)}{4^{2 q+1}}\left(1-\frac{1}{q+\kappa}\right) .
$$

For $\kappa=0.97$ this simple formula gives good numerical results for the basis integrals with an accuracy better than $1 \%$ for all $q \geq 1$. For $q>3$ the relative accuracy is better than $10^{-3}$ (one per mille).

Another representation for the basis set of integrals can be obtained using the dispersion relation in configuration space. One obtains the relation

$$
K_{0}^{4}(x)=(2 \pi)^{3} \int_{16}^{\infty} \rho_{4}(s) K_{0}(\sqrt{s} x) d s
$$

with $\rho_{4}(s)$ being a spectral density for the three-loop water melon diagram. Note that such a representation is a basis for the sum rule calculations in configuration space both in two-dimensional [20] and four-dimensional space-time [24]. One obtains the following relation for the basis integrals in terms of the moments of the spectral density $\rho_{4}(s)$,

$$
I(q)=(2 \pi)^{3} 4^{q}(q !)^{2} \int_{16}^{\infty} \frac{\rho_{4}(s)}{s^{q+1}} d s .
$$


An efficient way of computing the spectral density of water melon diagrams has recently been developed [25]. However, the direct configuration space representation is the most convenient for a numerical evaluation. To emphasize this last remark, we give an asymptotic formula for the $\varepsilon$-expansion of a water melon diagram where $\ln (x)$ appears in the integrand. The asymptotic formula for the log-type integrals reads (where we changed our notation by introducing $\ln (4 x)$ for convenience)

$$
\begin{aligned}
L_{4}^{l}(q) & =\int_{0}^{\infty} K_{0}^{4}(x) x^{2 q+1} \ln (4 x) d x \\
& =\frac{\pi^{2} \Gamma(2 q)}{4^{2 q+1}} \Psi(2 q)\left(1-\frac{1}{q-1 / 2}+O\left(1 / q^{2}\right)\right)
\end{aligned}
$$

where $\Psi(x)=\Gamma^{\prime}(x) / \Gamma(x)$ is the logarithmic derivative of the $\Gamma$-function.

Modifying the last term by introducing a parameter $\kappa_{l}$ as before in Eq. (53) we find that the relation

$$
L_{4}^{l}(q)=\frac{\pi^{2} \Gamma(2 q)}{4^{2 q+1}} \Psi(2 q)\left(1-\frac{1}{q+\kappa_{l}}\right)
$$

with $\kappa_{l}=1.17$ gives good results for the basis log-type integrals with an accuracy better than $1 \%$ for all $q>3$. For $q>5$ the relative accuracy is better than $10^{-3}$. The leading order asymptotic formula presented in Eq. (57) is less precise for small $q$ than its analog in Eq. (53) in the previous case because the integrand in Eq. (56) is not positive for log-type integrals. It is also clear that an exact solution of the recurrence relations will be much more complicated than these simple asymptotic formulae. For large $q$ the numerical cancellation of terms with different transcendental structure up to very high significant figures will be quite dramatic.

The solution of the recurrence relations depends on the space-time dimensionality. In the case $D=3$ the structure of the recurrence relations drastically simplifies. The basis set of integrals in three-dimensional space-time can be written down explicitly. The propagator is

$$
D(x, m)=\frac{1}{4 \pi x} e^{-m x}
$$

which leads to an integral basis of the form

$$
H_{0}(q)=(2 \pi)^{4(\lambda+1)} \int_{0}^{\infty} D(x, m)^{4} x^{q+2} d x \rightarrow \frac{\pi^{2}}{4} \int_{0}^{\infty} e^{-4 x} x^{q-2} d x=\frac{\pi^{2}}{4^{q}} \Gamma(q-1)
$$


The special case $D=3$ can serve as a check of any general solution of the recurrence relations.

\section{2 $\quad N=3$ water melon: a standard sunset}

The case of a two-loop water melon (genuine sunset) is simple indeed and can be easily analyzed along the same lines. The corresponding basis set of configuration space integrals is quite analogous to the previous case and is simpler because it now includes only three McDonald functions,

$$
\begin{aligned}
& J_{n}(q)=\int_{0}^{\infty} K_{0}^{3-n}(x) K_{1}^{n}(x) x^{q} d x \\
& J_{n}^{l}(q)=\int_{0}^{\infty} K_{0}^{3-n}(x) K_{1}^{n}(x) x^{q} \ln x d x .
\end{aligned}
$$

The reduction to the basis set of integrals analogous to the case of three-loop water melons given in Eqs. (43) and (44) can be now readily obtained.

The basic initial integral (the basic sunset $B_{S}$ ) for the recurrence relation has the explicit form

$$
B_{S}=\int \frac{\tilde{\Pi}_{2}(p)}{p^{2}+M^{2}} d^{2} p
$$

with $\tilde{\Pi}_{2}(p)$ given by Eq. (47). Here $M$ is a mass of the third line which is kept different from the other two with masses $m$. By differentiating with respect to $M$, any positive power of propagator (and/or power of $x$ in configuration space representation) can be obtained. After changing the integration variable as in the preceding subsection one finds an explicit representation

$$
B_{S}=\int_{0}^{\infty} \frac{\xi d \xi}{4 m^{2} \sinh ^{2}(\xi / 2)+M^{2}}
$$

After a change of variables the integration can be done and can be reduced to a polylogarithm function. Namely, for $t=e^{-\xi}$ one has

$$
B_{S}=\int_{0}^{\infty} \frac{\xi d \xi}{4 m^{2} \sinh ^{2}(\xi / 2)+M^{2}}=-\frac{1}{m^{2}} \int_{0}^{1} \frac{\ln t d t}{1-2 \gamma t+t^{2}}
$$

where $\gamma=1-M^{2} / 2 m^{2}$. The last integral is evaluated to give

$$
\int_{0}^{1} \frac{\ln t d t}{1-2 \gamma t+t^{2}}=\frac{\operatorname{Li}_{2}\left(1 / t_{1}\right)-\mathrm{Li}_{2}\left(1 / t_{2}\right)}{t_{1}-t_{2}}
$$


where $t_{1,2}=\gamma \pm \sqrt{\gamma^{2}-1}$ and $\operatorname{Li}_{2}(z)$ is the dilogarithm function,

$$
\operatorname{Li}_{2}(z)=\sum_{k=1}^{\infty} \frac{z^{k}}{k^{2}}, \quad|z|<1 .
$$

The differentiation with respect to $M$ is now straightforward and can be performed with a symbolic manipulation program.

In the case $M=2 m$ the integration simplifies because the two independent parameters $M$ and $2 m$ on which the integrand depends, coincide (degenerate case). The integral is then reduced to a special case of Eq. (49). We have $\gamma=-1$ and

$$
\int_{0}^{1} \frac{\ln t d t}{(1+t)^{2}}=-\ln 2
$$

which leads to

$$
B_{S}(M=2 m)=\frac{\ln 2}{m^{2}}
$$

For the case $M=m$ the standard result - Clausen's polylogarithm $\mathrm{Cl}_{2}(\pi / 3)$ - is reproduced (see e.g. ref. [8]). Indeed, $\gamma=1 / 2$ and $t_{1,2}=\exp ( \pm i \pi / 3)$. Eq. (64) now becomes

$$
\int_{0}^{1} \frac{\ln t d t}{1-t+t^{2}}=-\frac{2}{\sqrt{3}} \operatorname{Im~Li}_{2}\left(e^{i \pi / 3}\right)
$$

and with using the definition of Clausen's polylogarithm, $\mathrm{Cl}_{2}(\theta)=\operatorname{ImLi}_{2}\left(e^{i \theta}\right)$ one finds

$$
B_{S}(M=m)=\frac{2}{m^{2} \sqrt{3}} \mathrm{Cl}_{2}\left(\frac{\pi}{3}\right)
$$

\subsection{Generalization to the spectacle topology}

In this subsection we give a formula for a more general topology when only one propagator is removed from the initial three-loop bubble diagram. In the original classification of ref. [7] these are class $E$ diagrams belonging to the spectacle topology. The formula obtained in this subsection is efficient for numerical integration though we did not find any analytical solution. The main obstacle of generalizing the configuration space technique to a general multi-loop diagram is the angular integration. The configuration space technique proved to be rather successful for general diagrams in the massless case [26] but it brings no essential simplification in 
the general massive case (see e.g. ref. [27]). However, for special configurations the angular integration can be explicitly performed with a reasonably simple integrand left for the radial integration. The diagrams of spectacle topology give an example of such a configuration.

The configuration space expression of a spectacle topology diagram written in a form suitable for our purpose is (see Fig. 2(a))

$$
\int D(x-y, m) D(x, m)^{2} d^{D} x D(y, m)^{2} d^{D} y .
$$

The key relation for a drastic simplification of the configuration space integral with the spectacle topology is the addition theorem for Bessel functions allowing one to perform some angular integration explicitly. One needs to integrate over the relative angle in the propagator $D(x-y, m)$. In the handbook of Gradshteyn and Rhyshik [17] one finds

$$
\frac{Z_{\nu}(m R)}{R^{\nu}}=2^{\nu} m^{-\nu} \Gamma(\nu) \sum_{k=0}^{\infty}(\nu+k) \frac{J_{\nu+k}(m \rho)}{\rho^{\nu}} \frac{Z_{\nu+k}(m r)}{r^{\nu}} C_{k}^{\nu}(\cos \varphi)
$$

where $C_{k}^{\nu}$ are the Gegenbauer polynomials, $Z$ is any of the Bessel functions $J, Y$, $H^{(1)}$ or $H^{(2)}$,

$$
R=\sqrt{r^{2}+\rho^{2}-2 r \rho \cos \varphi}
$$

and $r>\rho$. For $r<\rho$ the arguments of the Bessel functions on the right hand side of Eq. (70) should be interchanged. Writing $R=|r-\rho|$ we have for $r>\rho$

$$
\frac{Z_{\nu}(m|r-\rho|)}{|r-\rho|^{\nu}}=2^{\nu} m^{-\nu} \Gamma(\nu) \sum_{k=0}^{\infty}(\nu+k) \frac{J_{\nu+k}(m \rho)}{\rho^{\nu}} \frac{Z_{\nu+k}(m r)}{r^{\nu}} C_{k}^{\nu}(\cos \varphi)
$$

Using this relation for $Z=H^{(1)}$ and substituting $m=e^{i \pi / 2}$ for the purpose of analytic continuation in order to obtain a relation for the modified Bessel functions $K$ and $I$, we find

$$
\frac{K_{\lambda}(|r-\rho|)}{|r-\rho|^{\lambda}}=2^{\lambda} \Gamma(\lambda) \sum_{k=0}^{\infty}(\lambda+k) \frac{I_{\lambda+k}(\rho)}{\rho^{\lambda}} \frac{K_{\lambda+k}(r)}{r^{\lambda}} C_{k}^{\lambda}(\cos \varphi)
$$

where we have

$$
\begin{aligned}
K_{\lambda}(z) & =\frac{i \pi}{2} e^{\pi \lambda i / 2} H_{\lambda}^{(1)}(i z), \\
I_{\lambda}(z) & =e^{-\pi \lambda i / 2} J_{\lambda}\left(e^{\pi i / 2} z\right) \quad \text { for }-\pi<\arg z \leq \frac{\pi}{2} \\
I_{\lambda}(z) & =e^{3 \pi \lambda i / 2} J_{\lambda}\left(e^{-3 \pi i / 2} z\right) \quad \text { for } \frac{\pi}{2}<\arg z \leq \pi
\end{aligned}
$$


Using the orthogonality relations for Gegenbauer polynomials (see the Appendix), the sum disappears after integration over the relative angle and only one term contributes. We obtain

$$
\begin{aligned}
\int \frac{K_{\lambda}(|r-\rho|)}{|r-\rho|^{\lambda}} d \Omega_{\rho} & =2^{\lambda} \Gamma(\lambda) \sum_{k=0}^{\infty}(\lambda+k) \frac{I_{\lambda+k}(\rho)}{\rho^{\lambda}} \frac{K_{\lambda+k}(r)}{r^{\lambda}} \int C_{k}^{\lambda}(\cos \varphi) d \Omega_{\varphi} \\
& =2^{\lambda} \Gamma(\lambda) \lambda \frac{I_{\lambda}(\rho)}{\rho^{\lambda}} \frac{K_{\lambda}(r)}{r^{\lambda}} \frac{2 \pi^{\lambda+1}}{\Gamma(\lambda+1)} C_{0}^{\lambda}(1) \\
& =(2 \pi)^{\lambda+1} \frac{I_{\lambda}(\rho)}{\rho^{\lambda}} \frac{K_{\lambda}(r)}{r^{\lambda}}, \quad r>\rho,
\end{aligned}
$$

where the first equality is a consequence of the orthogonality relation with the trivial factor $C_{0}^{\lambda}(1)=1$. This result allows one to write down an expression for any spectacle-type diagram in the form of a two-fold integral with a simple integration measure

$$
\begin{aligned}
\int_{0}^{\infty} D(x, m)^{2} x^{2 \lambda+1} d x \int_{0}^{\infty} D(y, m)^{2} y^{2 \lambda+1} d y \times \\
\left(\frac{K_{\lambda}(x)}{x^{\lambda}} \frac{I_{\lambda}(y)}{y^{\lambda}} \theta(x-y)+\frac{K_{\lambda}(y)}{y^{\lambda}} \frac{I_{\lambda}(x)}{x^{\lambda}} \theta(y-x)\right)
\end{aligned}
$$

where $\theta(x)$ is the standard step-function distribution.

Note that the integration measure $D(x, m)^{2} x^{2 \lambda+1} d x$ allows one to perform the integration by using efficient integration routines for a numerical evaluation. The form of the weight function is close to $e^{-a x} x^{\alpha}$ which makes the use of Laguerre polynomials a convenient choice within the Gaussian numerical integration method. Any modified propagator (with any power of the denominator) can be used as a factor in the integration measure $D(x, m)^{2} x^{2 \lambda+1} d x$ which makes this representation universal and useful for the case of high powers of denominators of the lines associated with pairs $(x, 0)$ and $(y, 0)$ of space-time points. If the angular structure of the diagram is preserved, the generalization to higher loops in the expressions for the radial measures is straightforward.

As an illustration of this technique we present an example of the evaluation of a spectacle diagram. Consider an integer dimension space-time which, without loss of generality, can be chosen to be two-dimensional (an odd number of dimensions is 
trivial because the propagators degenerate to simple exponentials). The spectacletype three-loop diagram can be obtained in a closed form. Indeed, in the momentum space representation we have

$$
S(M)=\int \frac{\tilde{\Pi}_{2}(p)^{2}}{p^{2}+M^{2}} d^{2} p
$$

for the basic spectacle diagram $S$ with $\tilde{\Pi}_{2}(p)$ taken from Eq. (47) and the mass $M$ of the connecting propagator kept different. After the substitutions $p=2 m \sinh (\xi / 2)$ and $t=e^{-\xi}$ we have

$$
S(M)=\frac{1}{2 \pi m^{4}} \int_{0}^{1} \frac{t \ln ^{2} t d t}{\left(1-t^{2}\right)\left(1-2 \gamma t+t^{2}\right)} .
$$

Performing the integration we finally obtain

$$
S(M)=\frac{f\left(t_{1}\right)-f\left(t_{2}\right)}{t_{1}-t_{2}}
$$

with

$$
f(t)=\frac{8 t \mathrm{Li}_{3}(1 / t)-(t+7) \zeta(3)}{8 \pi m^{4}\left(t^{2}-1\right)}
$$

where $t_{1,2}=\gamma \pm \sqrt{\gamma^{2}-1}$ with $\gamma=1-M^{2} / 2 m^{2}$ as before. $\operatorname{Li}_{3}(z)$ is the trilogarithm function

$$
\operatorname{Li}_{3}(z)=\sum_{k=1}^{\infty} \frac{z^{k}}{k^{3}}, \quad|z|<1 .
$$

For $M=2 m$ the integral in Eq. (78) simplifies as in the case of the sunset diagram and one finds a simple answer in terms of the standard (in the present context) transcendental numbers $\ln 2$ and $\zeta(3)$,

$$
S(2 m)=\frac{1}{4 \pi m^{4}}\left(\frac{7}{8} \zeta(3)-\ln 2\right)
$$

For the actual value of the mass $M=m$ we obtain a result including the next Clausen polylogarithm $\mathrm{Cl}_{3}(2 \pi / 3)$. As one can conclude from this expression, the conjugate pair of the sixth order roots of unity, $\exp ( \pm 2 \pi i / 3)$ plays an important role in this case again in accordance with the general analysis of ref. [8]. The origin of the appearance of the the sixth order roots of unity as the parameters of the analytical expressions of the diagrams lies in the mismatch of masses along the lines 
of the diagrams. However, the exceptional case $M=2 m$, where one line has the double mass of the other lines (which results in the drastic simplification) also keeps us within the set of the sixth order roots of unity. The key parameter in this case is simply the natural number 1 which definitely is one of the sixth order roots of unity.

Turning to the configuration space representation we find

$$
\begin{aligned}
S(M)= & \int_{0}^{\infty} x K_{0}(m x)^{2} d x \int_{0}^{\infty} y K_{0}(m y)^{2} d y \times \\
& \left(K_{0}(M x) I_{0}(M y) \theta(x-y)+K_{0}(M y) I_{0}(M x) \theta(y-x)\right)
\end{aligned}
$$

for the basic spectacle diagram. An explicit numerical integration of Eq. (80) shows coincidence with the analytical result in Eq. (79) which we checked for arbitrary values of $M$. In this example the analytical result has a rather simple form which is not true if high powers of denominators enter. Then the corresponding one-loop insertions are rather cumbersome and an explicit integration in configuration space is more convenient.

As a last topic of this subsection we demonstrate how the square of Clausen's polylogarithm $\mathrm{Cl}_{2}(\pi / 3)^{2}$ can emerge at the level of spectacle topology diagrams. The transcendental number $\mathrm{Cl}_{2}(\pi / 3)^{2}$ characterizes the analytical results for three-loop bubbles. Its presence was discovered in the impressive treatise of David Broadhurst on the role of the sixth order roots of unity for the transcendentality structure of results for Feynman diagrams in quantum field theory [8].

We consider the spectacle diagram in the form shown in Fig. 2(b). Here the expression for the generalized middle line is a product of the one-loop propagator of Eq. (47) and the standard particle propagator. We express the one-loop propagator in Eq. (47) using a dispersion representation with the spectral density $\rho_{2}(s)$ and obtain

$$
\begin{aligned}
& \operatorname{Loop}(p, m) \times \operatorname{Propagator}(p, m)=\tilde{\Pi}_{2}(p) \frac{1}{p^{2}+m^{2}}=\frac{1}{p^{2}+m^{2}} \int_{4 m^{2}}^{\infty} \frac{\rho_{2}(s) d s}{s+p^{2}} \\
& =\int_{4 m^{2}}^{\infty} \frac{\rho_{2}(s) d s}{s-m^{2}}\left(\frac{1}{p^{2}+m^{2}}-\frac{1}{s+p^{2}}\right) .
\end{aligned}
$$

Taking only the first term (which is sufficient for obtaining the result we are looking 
for) one has

$$
I=\frac{1}{p^{2}+m^{2}} \int_{4 m^{2}}^{\infty} \frac{\rho_{2}(s) d s}{s-m^{2}}
$$

which leads to the sunset diagram after the two other line shown in Fig. 2(b) have been added with a normalization factor given by the integral. One factor $\mathrm{Cl}_{2}(\pi / 3)$ results from integrating the overall sunset diagram which is composed of the propagator $\left(p^{2}+m^{2}\right)^{-1}$ from Eq. (82) with the two other lines of the diagram shown in Fig. 2(b). The second factor $\mathrm{Cl}_{2}(\pi / 3)$ has to be found in the normalization factor given by the integral in Eq. (82). Note that the very structure of this contribution - the square of a number which first appeared at the lower loop level - suggests a hint for its search. It should emerge as an iteration of a lower order contribution in accordance with the iterative structure of the $R$-operation (see e.g. ref. [28]) which forms a general framework for the analysis of multiloop diagrams. The following consideration confirms this guess. Consider the quantity

$$
N=\int_{4 m^{2}}^{\infty} \frac{\rho_{2}(s) d s}{s-m^{2}}
$$

and take $\rho_{2}(s)$ to be the spectral density in $D$-dimensional space-time (see e.g. Eq. (65) of ref. [15]),

$$
\rho_{2}(s)=\frac{\left(s-4 m^{2}\right)^{\lambda-1 / 2}}{2^{4 \lambda+1} \pi^{\lambda+1 / 2} \Gamma(\lambda+1 / 2) \sqrt{s}}, \quad \sqrt{s}>2 m .
$$

Now consider a first order contribution of the expansion in $\varepsilon$ near the space-time dimension $D=2$. The expansion in $\lambda=-\varepsilon$ results in

$$
\frac{\left(s-4 m^{2}\right)^{-\varepsilon-1 / 2}}{\mu^{2 \varepsilon} \sqrt{s}}=\frac{1}{\sqrt{s\left(s-4 m^{2}\right)}}\left(1-\varepsilon \ln \left(\frac{s-4 m^{2}}{\mu^{2}}\right)+O\left(\varepsilon^{2}\right)\right),
$$

so the relevant first order term in $\varepsilon$ is

$$
\Delta_{\varepsilon} \rho_{2}(s)=-\frac{\ln \left(\left(s-4 m^{2}\right) / m^{2}\right)}{2 \pi \sqrt{s\left(s-4 m^{2}\right)}}
$$

where $\mu=m$ has been chosen for convenience. Now we change the variable according to

$$
\sqrt{s}=2 m \cosh (\xi / 2), \quad t=e^{-\xi}
$$


to obtain

$$
\Delta_{\varepsilon} \rho_{2}\left(4 m^{2} \cosh ^{2}(\xi / 2)\right)=\frac{(\ln t-2 \ln (1-t)) t}{2 \pi m^{2}\left(1-t^{2}\right)} .
$$

For the quantity in question we find

$$
\Delta_{\varepsilon} N=\int_{4 m^{2}}^{\infty} \frac{\Delta_{\varepsilon} \rho_{2}(s) d s}{s-m^{2}}=\frac{1}{2 \pi m^{2}} \int_{0}^{1} \frac{(\ln t-2 \ln (1-t)) d t}{1+t+t^{2}}
$$

The roots of the denominator of the integrand in Eq. (88) are now $t_{3,4}=\exp ( \pm 2 \pi i / 3)$ which again is a conjugate pair of the sixth order roots of unity. After integrating this equation we readily find

$$
\begin{aligned}
\Delta_{\varepsilon} N & =\frac{1}{2 \pi m^{2} \sqrt{3}}\left(\operatorname{Im}\left(\operatorname{Li}_{2}\left(e^{2 i \pi / 3}\right)-\operatorname{Li}_{2}\left(e^{-2 i \pi / 3}\right)\right)-\frac{\pi}{3} \ln 3\right) \\
& =-\frac{1}{\pi m^{2} \sqrt{3}}\left(\mathrm{Cl}_{2}\left(\frac{2 \pi}{3}\right)-\frac{\pi}{6} \ln 3\right) .
\end{aligned}
$$

Using the relation

$$
\mathrm{Cl}_{2}\left(\frac{2 \pi}{3}\right)=\frac{2}{3} \mathrm{Cl}_{2}\left(\frac{\pi}{3}\right)
$$

we finally obtain

$$
\Delta_{\varepsilon} N=\frac{2}{3 \pi m^{2} \sqrt{3}}\left(\mathrm{Cl}_{2}\left(\frac{\pi}{3}\right)-\frac{\pi}{4} \ln 3\right) .
$$

Therefore, in the first order of the $\varepsilon$ expansion of the spectacle diagram we found this remarkable contribution proportional to $\mathrm{Cl}_{2}(\pi / 3)^{2}$. In our calculation it emerges naturally as the iteration of the lower order term. Originally this contribution had been guessed and confirmed in ref. [8] by a direct numerical computation of the finite part of the general three-loop bubble in four-dimensional space-time.

Note also that besides $B_{4}$ and $\mathrm{Cl}_{2}(\pi / 3)^{2}$ there is one more nontrivial transcendental number necessary for the analytical computation of the finite part of the general three-loop bubble in four-dimensional space-time as has been proven in ref. [8]. This number was chosen in ref. [8] as a two dimensional nested sum of the form

$$
V_{3,1}=\sum_{m>n>0} \frac{(-1)^{m} \cos (2 \pi n / 3)}{m^{3} n} .
$$

The analogous contribution may also appear in the first order $\varepsilon$-expansion of the spectacle diagram. We hope to devote a separate publication to this point. 
Therefore there are solid arguments that all basic transcendental numbers necessary for the analytical computation of three-loop massive bubbles can be found at the level of much simpler topology than the general tetrahedron. One may need only spectacle diagrams as the largest set to find all necessary transcendental numbers. These discoveries lead us to a conjecture about the extension of recurrence relations for three-loop bubbles beyond those found within the integration by part technique. Indeed, if all results can be obtained within the spectacle topology only, it looks plausible that there is a procedure that can perform a reduction of the general tetrahedron diagram to a simpler set where the spectacle topology is the most complicated one. The manifest form of this procedure is not known at present.

\section{Conclusion}

We have formulated a new representation for some of the massive three-loop vacuum diagrams with the simple water melon topology. We have obtained numerical values for some master integrals within our representation. The computation of diagrams with large powers of propagators is reduced to a linear (one-parameter) set of basis integrals. Our representation is simple and provides a tool for an efficient numerical evaluation of such diagrams. We have given asymptotic estimates which provide an accuracy better than one per mille for all basis integrals with $q>3$ in the case of the leading order of $\varepsilon$-expansion and with $q>5$ in the case of the first order of $\varepsilon$-expansion. The generalization to higher loops is straightforward. We gave strong arguments that all nontrivial transcendental numbers necessary for the computation of three-loop bubbles (which were identified in ref. [8] by using a high precision numerical integration) already appears at the level of spectacle topology diagrams.

As a by-product of our analysis we obtained analytical results for a number of definite integrals involving products of four McDonald functions of different orders with powers and logarithms (Sec. 3). 


\section{Acknowledgments}

We are indebted to K.G. Chetyrkin and D. Broadhurst for discussions, constructive criticism, remarks on the literature and friendly advice. A.A. Pivovarov thanks P.A. Baikov for an illuminating discussion of the present status of the optimization of recurrence relations under study by him. The work is supported in part by the Volkswagen Foundation under contract No. I/73611. A.A. Pivovarov is supported in part by the Russian Fund for Basic Research under contracts Nos. 97-02-17065

and 99-01-00091. S. Groote gratefully acknowledges a grant given by the Max Kade Foundation.

\section{Appendix}

We give some formulae for dealing with Gegenbauer polynomials in Sec. 5.4 that can be found e.g. in refs. [17, 26, 29]. The Gegenbauer polynomials obey the orthogonality relations

$$
\int C_{m}^{\lambda}\left(\hat{x}_{1} \cdot \hat{x}_{2}\right) C_{n}^{\lambda}\left(\hat{x}_{2} \cdot \hat{x}_{3}\right) d \hat{x}_{2}=\frac{\lambda}{n+\lambda} \delta_{m n} C_{n}^{\lambda}\left(\hat{x}_{1} \cdot \hat{x}_{3}\right) \quad\left(\int d \hat{x}_{2}=1\right)
$$

or, written in another form,

$$
\int C_{j_{1}}^{\lambda}(\hat{a} \cdot \hat{b}) C_{j_{2}}^{\lambda}(\hat{b} \cdot \hat{c}) d \Omega_{b}=\delta_{j_{1} j_{2}} \frac{2 \pi^{\lambda+1}}{\Gamma(\lambda+1)} \frac{\lambda}{j_{1}+\lambda} C_{j_{1}}^{\lambda}(\hat{a} \cdot \hat{c}) .
$$

In particular, we have $C_{0}^{\lambda}(x)=1, C_{1}^{\lambda}(x)=2 \lambda x$ and

$$
(j+1) C_{j+1}^{\lambda}(x)=2(j+\lambda) x C_{j}^{\lambda}(x)-(j+2 \lambda-1) C_{j-1}^{\lambda}(x)
$$

together with

$$
C_{j}^{\lambda}(1)=\frac{\Gamma(j+2 \lambda)}{j ! \Gamma(2 \lambda)} .
$$

The characteristic polynomial is given by

$$
\left(t^{2}-2 t x+1\right)^{-\lambda}=\sum_{j=0}^{\infty} t^{j} C_{j}^{\lambda}(x)
$$




\section{References}

[1] P. Langacker (ed.), "Precision Tests of the Standard Electroweak Model", World Scientific, 1995.

[2] L. Avdeev, J. Fleischer, S. Mikhailov and O. Tarasov, Phys. Lett. 336 B (1994) 560; 349 B (1995) 597(E);

J. Fleischer and O. Tarasov, Nucl. Phys. (Proc. Supp.) B37 (1994) 115

[3] K.G. Chetyrkin, J.H. Kühn and M. Steinhauser, Phys. Lett. 351 B (1995) 331

[4] K.G. Chetyrkin, J.H. Kühn and A. Kwiatkowski, Phys. Rep. 277 (1996) 189

[5] K.G. Chetyrkin and F.V. Tkachov, Nucl. Phys. B192 (1981) 159;

F.V. Tkachov, Phys. Lett. 100 B (1981) 65

[6] D.J. Broadhurst, Z. Phys. C54 (1992) 599.

[7] L.V. Avdeev, Comput. Phys. Commun. 98 (1996) 15

[8] D.J. Broadhurst, Eur. Phys. J. C8 (1999) 311

[9] K.G. Chetyrkin, private communication

[10] K.G. Chetyrkin, J.H. Kühn and M. Steinhauser, Phys. Lett. 371 B (1996) 93

[11] K.G. Chetyrkin, J.H. Kühn and M. Steinhauser, Nucl. Phys. B482 (1996) 213

[12] J.H. Kühn, A.A. Penin and A.A. Pivovarov, Nucl. Phys. B534 (1998) 356;

A.A. Penin and A.A. Pivovarov, Phys. Lett. 435 B (1998) 413;

Nucl. Phys. B549 (1999) 217

[13] K.G. Chetyrkin, J.H. Kühn and M. Steinhauser, Nucl. Phys. B505 (1997) 40

[14] P.A. Baikov, Phys. Lett. 385 B (1996) 404

[15] S. Groote, J.G. Körner and A.A. Pivovarov, Nucl. Phys. B542 (1999) 515

[16] G.N. Watson, "Theory of Bessel functions", Cambridge, 1944 
[17] I.S. Gradshteyn and I.M. Ryzhik, "Tables of integrals, series, and products", Academic Press, 1994

[18] D.J. Broadhurst, Z. Phys. C47 (1990) 115

[19] K.G. Chetyrkin, in "New computing techniques in physics research", Proceedings of the 3rd AIHENP Workshop, Oberammergau, Germany, October 4-8, 1993, by K.H. Becks and D. Perret-Gallix (eds.), World Scientific Singapore (1994) $664 \mathrm{p}$.

[20] A.A. Pivovarov, N.N. Tavkhelidze and V.F. Tokarev, Phys. Lett. 132 B (1983) 402;

A.A. Pivovarov and V.F. Tokarev, Yad. Fiz. 41 (1985) 524

[21] M. Steinhauser, PhD thesis, Universität Karlsruhe (Shaker Verlag, Aachen, 1996).

[22] P.A. Baikov, M. Steinhauser, Comput. Phys. Commun. 115 (1998) 161

[23] A.P. Prudnikov, Yu.A. Brychkov and O.I. Marichev, "Integrals and Series", Vol. 2, Gordon and Breach, New York, 1990

[24] K.G. Chetyrkin and A.A. Pivovarov, Nuovo Cim. 100 A (1988) 899

[25] S. Groote, J.G. Körner and A.A. Pivovarov, Phys. Lett. 443 B (1998) 269

[26] K.G. Chetyrkin, A.L. Kataev and F.V. Tkachev, Nucl. Phys. B174 (1980) 345

[27] E. Mendels, Nuovo Cim. 45 A (1978) 87

[28] N.N. Bogoliubov and D.V. Shirkov, "Quantum fields", Benjamin, 1983

[29] A.E. Terrano, Phys. Lett. 93 B (1980) 424 